\title{
Medical Image of the Week: Prozac Eyes
}

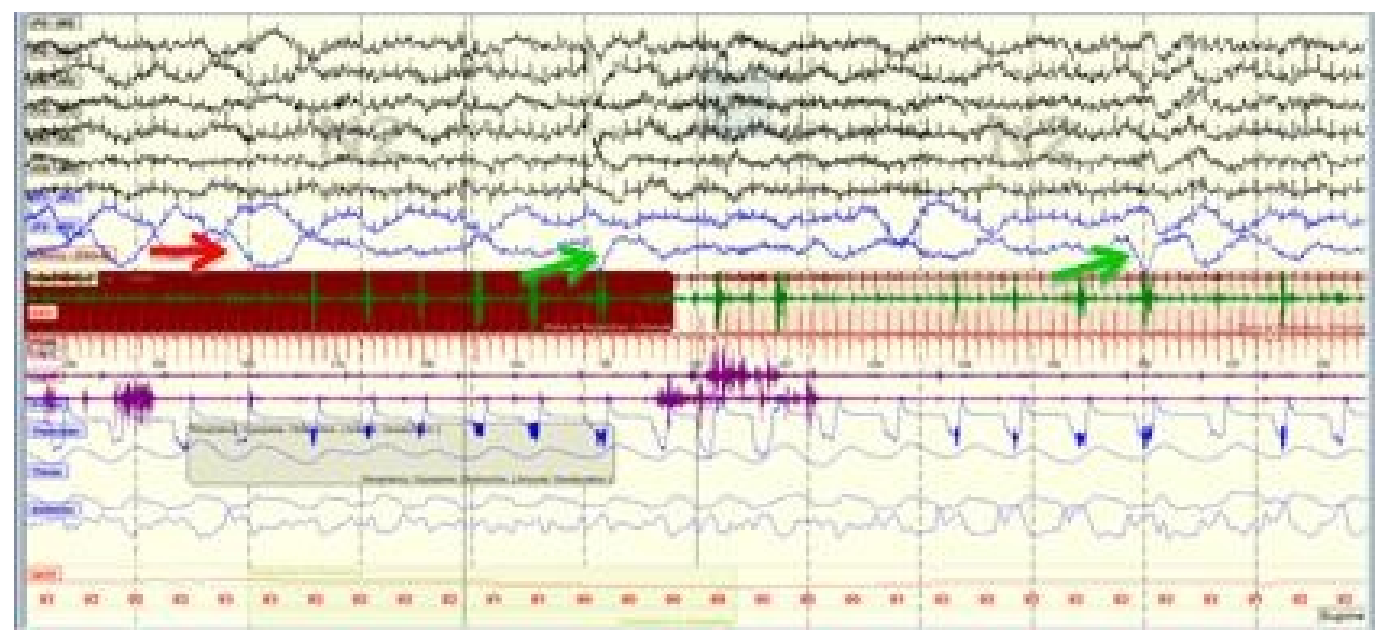

Figure 1. 60-second epoch shows the slow rolling eye movements (red arrow) and rapid eye movements (green arrows) seen during NREM stage 2 sleep.

A 59-year-old man with a past medical history significant for hypertension, obesity and depression underwent an overnight polysomnogram for high clinical suspicion for obstructive sleep apnea. His current medications include doxepin, fluoxetine, bupropion, ambien and amlodipine. A snapshot during NREM sleep is shown (Figure 1).

Fluoxetine (Prozac $®$ ) is a potent selective serotonin reuptake inhibitor (SSRI)."Omnipause" neurons in the brainstem inhibit saccadic eye movements. NREM eye movements result from the potentiation of serotonergic neurons that inhibit these neurons (1). These eye movements occur during all stages of NREM sleep. These atypical eye movements have been reported to be present with a lower incidence with use of other antidepressants, benzodiazepines and neuroleptics and they tend to persist even after discontinuation of the medication (2). The clinical significance of these eye movements is unknown.

Safal Shetty MD, Sarah Patel MD, Kenneth S. Knox MD

Section of Pulmonary, Allergy, Critical Care \& Sleep Medicine

Banner University Medical Center

Tucson, AZ USA

\section{References}

1. Schenck $\mathrm{CH}$, Mahowald MW, Kim SW, O'Connor KA, Hurwitz TD.Prominent eye movements during NREM sleep and REM sleep behavior disorder associated with fluoxetine treatment of depression and obsessive-compulsive disorder. Sleep. 1992;15(3):226-35. [PubMed]

2. Geyer JD, Carney PR, Dillard SC, Davis L, Ward LC. Antidepressant medications, neuroleptics, and prominent eye movements during NREM sleep. J Clin Neurophysiol. 2009;26(1):39-44. [CrossRef] [PubMed] 